\title{
Impacts of Oxidative Stress and Antioxidants on Semen Functions
}

\author{
Amrit Kaur Bansal and G. S. Bilaspuri \\ Department of Veterinary Gynaecology and Obstetrics, Guru Angad Dev Veterinary and Animal Sciences University, \\ Ludhiana 141004, India \\ Correspondence should be addressed to Amrit Kaur Bansal, bansal2amrit@yahoo.co.in
}

Received 23 April 2010; Revised 2 June 2010; Accepted 16 August 2010

Academic Editor: Ali Honaramooz

Copyright ( 2011 A. K. Bansal and G. S. Bilaspuri. This is an open access article distributed under the Creative Commons Attribution License, which permits unrestricted use, distribution, and reproduction in any medium, provided the original work is properly cited.

\begin{abstract}
Oxidative stress (OS) has been considered a major contributory factor to the infertility. Oxidative stress is the result of imbalance between the reactive oxygen species (ROS) and antioxidants in the body which can lead to sperm damage, deformity, and eventually male infertility. Although high concentrations of the ROS cause sperm pathology (ATP depletion) leading to insufficient axonemal phosphorylation, lipid peroxidation, and loss of motility and viability but, many evidences demonstrate that low and controlled concentrations of these ROS play an important role in sperm physiological processes such as capacitation, acrosome reaction, and signaling processes to ensure fertilization. The supplementation of a cryopreservation extender with antioxidant has been shown to provide a cryoprotective effect on mammalian sperm quality. This paper reviews the impacts of oxidative stress and reactive oxygen species on spermatozoa functions, causes of ROS generation, and antioxidative strategies to reduce OS. In addition, we also highlight the emerging concept of utilizing OS as a tool of contraception.
\end{abstract}

\section{Introduction}

Gametes are susceptible to reactive oxygen species (ROS) attack. When manipulated in vitro during assisted reproductive techniques, these cells run the risk of generating and being exposed to supra-physiological level of ROS [1]. Defective sperm functions are the most prevalent causes of male infertility and a difficult condition to treat [2]. Many environmental, physiological, and genetic factors have been implicated in the poor sperm functions and infertility [3-6]. Thus, it is very important to identify the factors/conditions which affect normal sperm functions. Among various causes, oxidative stress (OS) has been attributed to affect the fertility status and physiology of spermatozoa [7]. The term oxidative stress is generally applied when oxidants outnumber antioxidants [1]. The imbalance between the production of reactive oxygen species (ROS) and a biological systems ability to readily detoxify the reactive intermediates or easily repair the resulting damage is known as oxidative stress [8]. The main destructive aspects of oxidative stress are the production of ROS, which include free radicals and peroxides [9].

The production of ROS by sperm is a normal physiological process, but an imbalance between ROS generation and scavenging activity is detrimental to the sperm and associated with male infertility [10]. Physiological levels of ROS influence and mediate the gametes [11-13] and crucial reproductive processes, such as sperm-oocyte interactions [14], implantation and early embryo development [15].

Against ROS attack, sperm cells are well equipped with a powerful defense system of antioxidants [2]. Antioxidants are the main defense factors against oxidative stress induced by free radicals [2]. This article reviews the impact of oxidative stress and reactive oxygen species (ROS) on spermatozoal functions, causes of ROS generation, and antioxidative strategies to reduce the oxidative stress. In addition, the significance of antioxidants in reproduction and oxidative stress as a contraceptive measure will be reviewed.

\section{Oxidative Stress}

One of the most important factors contributing to poor quality semen has been reported to be oxidative stress [16]. Oxidative stress is a condition associated with an increased rate of cellular damage induced by oxygen and oxygen derived oxidants commonly known as ROS [17]. 
Uncontrolled production of ROS that exceeds the antioxidant capacity of the seminal plasma leads to oxidative stress (OS) which is harmful to spermatozoa [18]. All cellular components including lipids, proteins, nucleic acids, and sugars are potential targets of oxidative stress [7].

\section{Free Radicals}

Free radicals are short lived reactive chemical intermediates, which contain one or more unpaired electrons $[19,20]$. They induce cellular damages when they pass this unpaired electron onto nearby cellular structures, resulting in oxidation of cell membrane lipids, amino acids in proteins or within nucleic acids [21]. Free radicals are also known as a necessary evil for intracellular signaling involved in the normal process of cell proliferation, differentiation, and migration [22-24]. In the reproductive tract, free radicals also play a dual role and can modulate various reproductive functions [1]. Excess of free radicals generation frequently involves an error in spermiogenesis resulting in the release of spermatozoa from the germinal epithelium exhibiting abnormally high levels of cytoplasmic retention [20].

\section{Reactive Oxygen Species (ROS)}

ROS are formed as necessary by-products during the normal enzymatic reactions of inter- and intracellular signaling. Mammalian spermatozoa represent a growing list of cell types that exhibit a capacity to generate ROS when incubated under aerobic conditions, such as, hydrogen peroxide $\left(\mathrm{H}_{2} \mathrm{O}_{2}\right)$, the superoxide anion $\left({ }^{\circ} \mathrm{O}_{2}{ }^{-}\right)$, the hydroxyl radical $\left(\mathrm{OH}_{.}\right)$, and hypochlorite radical $\left(\mathrm{OHCl}_{\text {. }}\right)$. Due to their highly reactive nature, ROS can combine readily with other molecules, directly causing oxidation that can lead to structural and functional changes and result in cellular damage [25-27].

\section{Types of ROS}

ROS represent a broad category of molecules that indicate the collection of radicals (hydroxyl ion, superoxide, nitric oxide, peroxyl, etc.) and nonradicals (ozone, single oxygen, lipid peroxides, hydrogen peroxide) and oxygen derivatives [28]. Reactive nitrogen species (nitrous oxide, peroxynitrite, nitroxyl ion, etc.) are free nitrogen radicals and considered a subclass of ROS [29, 30]. Nitric oxide (NO) has been shown to have detrimental effects on normal sperm functions inhibiting both motility and sperm competence for zona binding [7].

\section{Origin of ROS in Male Reproductive System/Sources of ROS}

In male, two ROS generating systems are possibly involved, a hypothetical NADH oxidase at the level of sperm membrane and low sperm diphorase (mitochondrial NADH-dependent oxidoreductase) [31]. In bovine semen, ROS are generated primarily by dead spermatozoa via an aromatic amino acid oxidase catalyzed reaction [32].

Leukocytes and immature spermatozoa are the two main sources of ROS [33]. Leukocytes particularly neutrophils and macrophages have been associated with excessive ROS production and they ultimately cause sperm dysfunction $[21,34-40]$.

Two of the main factors contributing to ROS accumulation in vitro are the absence of endogenous defense mechanism and second exposure of gametes and embryos to various manipulation techniques as well as environment that can lead to generation of oxidative stress [1]. Levels of ROS may rarely fluctuate within a fertile individual, but, do not affect sperm concentration and motility. This may be due to the presence of adequate antioxidant defense mechanisms in the healthy individuals. The fluctuations in ROS levels might be due to transient subclinical infection and transient abnormalities in spermatogenesis such as, retention of cytoplasm or periodic presence of abnormal spermatozoa in semen [18].

\section{Positive and Negative Effects of ROS}

The production of ROS is a normal physiological process but an imbalance between ROS generation and scavenging activity is detrimental to the sperm and associated with male infertility [10]. ROS generated by spermatozoa play an important role in normal physiological processes such as, sperm capacitation, acrosome reaction, maintenance of fertilizing ability, and stabilization of the mitochondrial capsule in the mid-piece in bovine [7, 41, 42]. Controlled generation of ROS may function as signaling molecules (second messengers) in many different cell types; they are important mediators of sperm functions. Evidences have been reported that especially superoxide anion $\left({ }^{\bullet} \mathrm{O}_{2}\right)$ is required for the late stage of embryo development such as, two germ cell layers and egg cylinder [43]. Although a significant negative correlation between ROS and IVF fertilization rate has been found [27], yet, controlled generation of ROS has shown to be essential for the development of capacitation and hyperactivation [44]; the two processes of sperm that are necessary to ensure fertilization. In vivo physiological concentrations of ROS are involved in providing membrane fluidity, maintaining the fertilizing ability and acrosome reaction of sperm [16]. The maintenance of a suitable ROS level is, therefore, essential for adequate sperm functionality. ROS cause adverse effects on the sperm plasma membrane, DNA, and physiological processes, thereby, affecting the quality of spermatozoa. The axosome and associated dense fibers of the mid-piece in sperm are covered by mitochondria that generate energy from intracellular stores of ATP depletion [45]. Excessive ROS impairs motility and capacity of fertilization. Additionally, cold shock arising from other stress plays an important role in the molding of membranes by determining their sol gel balance and the dynamic status that affects the fusion of the plasma membrane of the male and female gametes [46]. The assumption that ROS can influence male fertility has received substantial scientific support [27]. 
Mammalian spermatozoal membranes are rich in polyunsaturated fatty acids (PUFAs) and are sensitive to oxygen-induced damage mediated by lipid peroxidation, and, thus are sensitive to ROS attack which results in decreased sperm motility, presumably by a rapid loss of intracellular ATP leading to axonemal damage, decreased sperm viability, and increased mid-piece sperm morphological defects with deleterious effects on sperm capacitation and acrosome reaction $[2,47]$. Thus ROS are independent markers of male factor infertility [17].

\section{Lipid Peroxidation}

The mechanism of ROS-induced damage to spermatozoa includes an oxidative attack on the sperm membrane lipids leading to initiation of lipid peroxidation (LPO) cascade [10].

Mammalian spermatozoa are known to be susceptible to loss of motility in the exogenous oxidant, as a consequence of LPO [48]. The susceptibility of ruminant spermatozoa to oxidative stress is a consequence of the abundance of PUFAs in sperm plasma membrane, the presence of which gives the membranes fluidity and flexibility which help the sperm to engage in membrane fusion events associated with the fertilization. Unfortunately, the presence of double bonds in these molecules makes them vulnerable to free radicals attack and the initiation of LPO cascade. This results in a subsequent loss in membrane and morphological integrity, impaired cell functions, along with impaired sperm motility and induction of sperm apoptosis [16].

Concerning the chemistry of LPO in spermatozoa, it implies that once this process has been initiated, its propagation is impeded, leading to accumulation of lipid peroxides in the sperm plasma membrane [10]. Supplementation of transition metal ions such as $\mathrm{Fe}^{2+}$ to the sperm suspension results in a sudden acceleration of LPO and loss of sperm functions such as motility and viability [49]. The key intermediates in spontaneous LPO are the lipid hydroperoxides generated by a chain reaction initiated and their subsequent utilization. Peroxidation of PUFAs in sperm cell membrane is an autocatalytic, self-propagating reaction, which can give rise to cell dysfunction associated with the loss of membrane functions and integrity.

\section{Lipid Peroxidation-Detrimental Effects on Sperm Functions}

Lipid peroxides are spontaneously generated in the sperm plasma membrane and are released by the action of phospholipase $A_{2}$. They are capable of inducing DNA damage and decrease in fertility during storage of semen [50]. The peroxides are generally associated with decreased sperm functions and viability, but, have also a significant enhancing effect on the ability of spermatozoa to bind with homologous and heterologous zona pellucida [51].

\section{Measurement of LPO-Thiobarbituric Acid Assay}

Malondialdehyde (MDA) is an end product of LPO which is measured through thiobarbituric acid (TBA) assay [20]. TBA reactive substances (TBARS) are mainly formed during the determination of LPO in vitro [52]. In many studies, TBARS level, as an indicator of LPO, was increased in frozen thawed bull sperm, but, not in cooled sperm [53]. Malondialdehyde (MDA) levels do not differ in fresh and frozen semen in normal men [54]. In bull semen, spontaneous LPO is measured by the endogenous lipid peroxides [55].

\section{Strategies to Reduce Oxidative Stress}

11.1. Antioxidants. Spermatozoa are protected by various antioxidants and antioxidant enzymes in the seminal plasma or in spermatozoa itself to prevent oxidative damage [56]. An antioxidant that reduces oxidative stress and improves sperm motility could be useful in the management of male infertility [57]. Antioxidants are the agents, which break the oxidative chain reaction, thereby, reduce the oxidative stress $[58,59])$. Vitamin $\mathrm{E}$ (antioxidant) may directly quench the free radicals such as peroxyl and alkoxyl (ROO.) generated during ferrous ascorbate-induced LPO, thus it is suggested as major chain breaking antioxidant [60]. Antioxidants, in general, are the compounds and reactions which dispose, scavenge, and suppress the formation of ROS, or oppose their actions. $\mathrm{Mn}^{2+}$ enhances sperm motility, viability, capacitation and acrosome reaction by decreasing the oxidative stress $[57,61]$. Extracellular addition of $\mathrm{Mn}^{2+}$ ions also enhances the level of cAMP by stimulating $\mathrm{Ca}^{2+}$ or $\mathrm{Mg}^{2+}$ ATPase which leads to activation of calcium channel opening, thereby depositing more $\mathrm{Ca}_{i}^{2+}$. Thus, $\mathrm{Mn}^{2+}$ promotes the acrosome reaction [56]. Thiol groups also play an important role in detoxification and antioxidation of ROS, besides maintaining the intracellular redox status. These groups serve as defense mechanisms of sperm cells to fight against oxidative stress [62]. A variety of biological and chemical antioxidants that attack ROS and LPO are presently under investigation [2]. Recent studies demonstrate that supplementation of cryopreservation extenders with antioxidants has been shown to provide a cryoprotective effect on bull, ram, goat, boar, canine, and human sperm quality, thus improving semen parameters, for example, sperm motility, membrane integrity after thawing [16]. Supplementation with these antioxidants prior to the cryopreservation process may be recommended to facilitate the enhancement of sperm cryopreservation technique for the goat breeding industry [16]. Supplementation with antioxidants during IVF procedures impaired sperm quality, normal pronuclear formation, and embryo development to the blastocyst stage [41].

Enzymatic Antioxidants. Enzymatic antioxidants are also known as natural antioxidants; they neutralize excess ROS and prevent it from damaging the cellular structure. Enzymatic antioxidants are composed of superoxide dismutase (SOD), catalase, glutathione peroxidase (GPx), and 
glutathione reductase (GR) which also causes reduction of hydrogen peroxides to water and alcohol [27]. SOD spontaneously dismutase superoxide anion $\left(\mathrm{O}_{2}^{-}{ }^{\bullet}\right)$ to form $\mathrm{O}_{2}$ and $\mathrm{H}_{2} \mathrm{O}_{2}$ while catalase converts $\mathrm{H}_{2} \mathrm{O}_{2}$ to $\mathrm{O}_{2}$ and $\mathrm{H}_{2} \mathrm{O}$ as shown in following equation [2].

$$
\begin{gathered}
2\left(\mathrm{O}_{2}^{-} \cdot\right)+2 \mathrm{H}^{+} \stackrel{\mathrm{SOD}}{\longrightarrow} \mathrm{H}_{2} \mathrm{O}_{2}+\mathrm{O}_{2}, \\
\mathrm{H}_{2} \mathrm{O}_{2} \stackrel{\text { Catalase }}{\longrightarrow} \mathrm{H}_{2} \mathrm{O}+\frac{1}{2} \mathrm{O}_{2} .
\end{gathered}
$$

SOD protects spermatozoa against spontaneous $\mathrm{O}_{2}$ toxicity and LPO [2]. SOD and catalase also remove $\mathrm{O}_{2}^{-} \cdot{ }_{2}$ generated by NADPH oxidase in neutrophils and play a major role in decreasing LPO and protecting spermatozoa against oxidative damage [2]. Catalse presence in sperm has been demonstrated for ram and cattle and it has a potential role in ageing process and control of oxidative stress in cells, mainly resulting from $\mathrm{H}_{2} \mathrm{O}_{2}$ [46].

Nonenzymatic Antioxidants. Nonenzymatic antioxidants are also known as synthetic antioxidants or dietary supplements. The body's complex antioxidant system is influenced by dietary intake of antioxidants, vitamins, and minerals such as vitamin $\mathrm{C}$, vitamin $\mathrm{E}$, zinc, taurine, hypotaurine, and glutathione [27].

Glutathione is a molecule found at $\mathrm{mM}$ level in a number of cells, able to react with many ROS directly [45]. GSH is also a cofactor for GSHPx that catlyzes the reduction of toxic $\mathrm{H}_{2} \mathrm{O}_{2}$ and other hydroperoxides, protecting the mammalian cells from oxidative stress [45]. Glutamine ( $5 \mathrm{mM})$ has been provided a cryoprotective effect by improving postthaw motility, membrane integrity, and catalse enzyme activity in ram semen [63].

Supplementation of inositol in the extender can improve the motility of frozen thawed bull sperm [16]. Inositol has cryoprotective and antioxidative properties resulting in higher antioxidant GSH activity, acrosome integrity, and intact morphological rates [16].

Cysteine is a low-molecular weight amino acid containing thiol; it is a precursor of intracellular glutathione[64]. It has been shown to penetrate the cell membrane easily, enhancing the intracellular GSH biosynthesis both in vivo and in vitro and protecting the membrane lipids and proteins due to indirect radical scavenging properties [37]. It is also thought that GSH synthesis under in vitro conditions may be impaired because of deficiency of cysteine in the media, due to its high instability and auto-oxidation to cysteine [45]. Cysteine has cryoprotective effect on the functional integrity of axosome and mitochondria improving postthawed sperm motility. It has been proved that thiols such as glutathione and cysteine prevented the loss of sperm motility in frozen thawed bull semen [64]. Cysteine has been shown to prevent the loss in motility of frozen thawed bull, ram, and goat semen and to improve viability, the chromatin structure, and membrane integrity of boar sperm during liquid preservation [64]. Cysteine has improved the porcine oocytes maturation and fertilization in vitro [37].

Trehalose or taurine, a sulfonic amino acid, acts as nonenzymatic scavenger that plays an important role in the protection of spermatozoa against ROS, in case of exposure to aerobic conditions and the freezing-thawing process [65]. A nonpermanent disaccharide has a protective action related both to osmotic effect and specific interactions with membrane phospholipids, rendering hypertonic media, causing cellular osmotic dehydration before freezing and then decreasing the amount of cell injury by its crystallization [46]. Trehalose performs better cryoprotection postthaw fertilizing ability in ram, bull, and mouse sperm due to diminished death and damage of sperm [46]. A recent study has demonstrated that antioxidant capacity of trehalsoe is observed upon the performance of incubation at $37^{\circ} \mathrm{C}$ for $3 \mathrm{~h}$ and no difference is obtained at $0 \mathrm{~h}$ postthawing of ram semen [46]. Tuarine displayed antioxidative properties by elevating catalse level in close association with superoxide dismutase concentration in ram, rabbit, and bull spermatozoa[65].

Hyaluronan, an essential component of the extracellular matrix and nonsulfated glycosaminoglycan, is involved in important physiological functions such as motility, capacitation of spermatozoa and preserve postthaw spermatozoa viability, and in vitro membrane stability [46]. Hyaluronan improves sperm motility, viability and membrane integrity after freezing and thawing procedure and decrease polyspermy with declining motility in humans and boars [46].

Bovine serum albumin (BSA) is known to eliminate free radicals generated by oxidative stress and protect membrane integrity of sperm cells from heat shock during freezingthawing of canine semen [64].

Carotenoids such as beta-carotene and lycopene are also important components of antioxidant defense. Beta carotenes protect the plasma membrane against LPO in rat [64].

Addition of antioxidants vitamin E, butylated hydroxytoluene (BHT), and Tempo to extended turkey semen improves sperm survival and membrane integrity and reduces the loss of motility after $48 \mathrm{~h}$ of storage [64].

Since long it has been known that supplementation of culture media with antioxidants such as, ROS scavengers, disulfide reducing, or divalent chelators prolongs the motility of reactivated bull spermatozoa after freezing and thawing [66]. It has been suggested that antioxidant therapy appears to be efficient not only in vitro but also in vivo [66].

Numerous antioxidants have proven beneficial in protecting damaging effects of ROS on sperm movement and against oxidative damage [67].

\section{Cryopreservation/Freezing Thawing-Oxidative Stress}

Semen cryopreservation is an important procedure which allows specific advantages to livestock industry [63]. The improvement of semen cryopreservation techniques requires in depth knowledge of the gamete physiology and the biochemical processes occurring during semen collection, processing, and freeze-thawing. Freezing/thawing of sperm sample is routinely performed in cattle breeding industries 
in order to perform artificial insemination. These procedures are known to produce ROS in sperm samples. During cryopreservation, semen is exposed to cold shock and atmospheric oxygen, which in turn increases the susceptibility to lipid peroxidation due to higher production of ROS [45]. As the sperm plasma membrane is one of the key structures affected by cryopreservation [22, 67], sperm cryopreservation and thawing is associated with increased ROS production and decreased antioxidant level. Both freezing and thawing cause tremendous alterations in cell water volume. Spermatozoa discard most of their cytoplasm during the terminal stages of differentiation and lack the significant cytoplasmic component containing antioxidants that counteract the damaging effect of ROS and LPO [46]. Due to this, spermatozoa are susceptible to LPO during cryopreservation and thawing [46], which confers considerable mechanical stress on the cell membrane [67]. It has been noted in humans that ROS level has a positive correlation with the extent of apoptotic sperms [68]. Despite recent morphological advances, cryopreservation exerts detrimental effects on spermatozoa that lead to a significant decrease in sperm viability and motility, and, ultimately in decreased cryopreserved sperm rates (CSR). The fertility potential of cryopreserved mammalian spermatozoa is lower than that of fresh sperm [69]. Frozen-thawed ram sperm demonstrates serious cryopreservation damage and thus a highly reduced fertilizing capacity [64]. Long-term (freezing) and short-term (liquid) storage of sperm may lead to membrane deterioration due to membrane phase transition occurring in the regions of highly specialized sperm plasma membrane [63]. Antioxidant capacity of semen may, however, be insufficient in preventing LPO during the freezing thawing process. The protective antioxidant systems in sperm are primarily of cytoplasmic origin and sperm discard most of their cytoplasm during terminal stages of differentiation [16]. Thus mammalian sperm lack a significant cytoplasmic component, which contains sufficient antioxidants to counteract the damaging effects of ROS and LPO. For this reason, sperm are susceptible to LPO during cryopreservation and thawing process [16]. The improvement of cryopreservation technique requires in depth knowledge of the gamete physiology and the biochemical processes occurring during semen collection, processing, and freezingthawing. Damage due to oxidative stress may be by passed by the inclusion of antioxidants prior to freezing processes [45].

Cryopreservation induces extensive biophysical and biochemical changes in the membrane of spermatozoa that ultimately decrease the fertility potential of the cells [70]. Procedure of cryopreservation increases premature capacitation of spermatozoa [65]. These alterations may not affect motility but reduces life span, ability to interact with the female reproductive tract and sperm fertility. Freezing and thawing processes also lead to the generation of reactive oxygen species (ROS) [45]. Excessive production of ROS during cryopreservation has been associated with the reduced postthaw motility, viability, membrane integrity, antioxidant status, and fertility and sperm functions. The postthaw motility of the cryopreserved buffalo semen is poor and the success rate of IVF with buffalo sperm is only $10 \%-$ $20 \%$ as compared to cattle which is $30 \%-35 \%$ [64].

\section{Oxidative Stress and Contraception}

Lipid peroxidation induced by $\mathrm{H}_{2} \mathrm{O}_{2}$ not only disrupts sperm motility, but, also impairs all the sperm functions which are dependent on the integrity of plasma membrane, including sperm-oocyte fusion and ability to undergo acrosomal exocytose. Such findings have raised the possibility that hydrogen peroxide or reagents producing them on contact with spermatozoa might be an effective way of contraception.

\section{Conclusions}

Evaluation of OS and the use of antioxidants are not routine in clinical practice. The immediate need is to simplify and validate the evaluation of ROS and OS status so that it can be performed routinely without the use of sophisticated equipment. Also, it is important to establish reference values for ROS above which antioxidants could be used for male infertility treatment. The dose and duration of these antioxidants should also be determined and standardized. With the increase in the use of ART procedure, there should be an effort to develop optimum combinations of antioxidants to supplement sperm preparation media. The proposal to use hydrogen peroxide producing agents as possible means of contraception warrants further investigation.

\section{References}

[1] S. S. du Plessis, K. Makker, N. R. Desai, and A. Agarwal, "Impact of oxidative stress on IVF," Expert Review of Obstetrics and Gynecology, vol. 3, no. 4, pp. 539-554, 2008.

[2] S. C. Sikka, "Oxidative stress and role of antioxidants in normal and abnormal sperm function," Frontiers in Bioscience, vol. 1, pp. e78-e86, 1996.

[3] I. D. Sharlip, J. P. Jarow, A. M. Belker et al., "Best practice policies for male infertility," Fertility and Sterility, vol. 77, no. 5, pp. 873-882, 2002.

[4] M. F. Vine, "Smoking and male reproduction: a review," International Journal of Andrology, vol. 19, pp. 323-337, 1996.

[5] J. Auger, F. Eustache, A. G. Andersen et al., "Sperm morphological defects related to environment, lifestyle and medical history of 1001 male partners of pregnant women from four European cities," Human Reproduction, vol. 16, no. 12, pp. 2710-2717, 2001.

[6] S. Kenkel, C. Rolf, and E. Nieschlag, "Occupational risks for male fertility: an analysis of patients attending a tertiary referral centre," International Journal of Andrology, vol. 24, no. 6, pp. 318-326, 2001.

[7] A. Agarwal, K. Makker, and R. Sharma, "Clinical relevance of oxidative stress in male factor infertility: an update," American Journal of Reproductive Immunology, vol. 59, no. 1, pp. 2-11, 2008.

[8] A. Agarwal, R. A. Saleh, and M. A. Bedaiwy, "Role of reactive oxygen species in the pathophysiology of human reproduction," Fertility and Sterility, vol. 79, no. 4, pp. 829843, 2003.

[9] M. Valko, H. Morris, and M. T. D. Cronin, "Metals, toxicity and oxidative stress," Current Medicinal Chemistry, vol. 12, no. 10, pp. 1161-1208, 2005.

[10] R. K. Sharma and A. Agarwal, "Role of reactive oxygen species in male infertility," Urology, vol. 48, no. 6, pp. 835-850, 1996. 
[11] C. Gagnon, A. Iwasaki, E. De Lamirande, and N. Kovalski, "Reactive oxygen species and human spermatozoa," Annals of the New York Academy of Sciences, vol. 637, pp. 436-444, 1991.

[12] R. J. Aitken, "Molecular mechanisms regulating human sperm function," Molecular Human Reproduction, vol. 3, no. 3, pp. 169-173, 1997.

[13] M. Attaran, E. Pasqualotto, T. Falcone et al., "The effect of follicular fluid reactive oxygen species on the outcome of in vitro fertilization," International Journal of Fertility and Women's Medicine, vol. 45, no. 5, pp. 314-320, 2000.

[14] E. de Lamirande, P. Leclerc, and C. Gagnon, "Capacitation as a regulatory event that primes spermatozoa for the acrosome reaction and fertilization," Molecular Human Reproduction, vol. 3, no. 3, pp. 175-194, 1997.

[15] D. Sakkas, F. Urner, D. Bizzaro et al., "Sperm nuclear DNA damage and altered chromatin structure: effect on fertilization and embryo development," Human Reproduction, vol. 13, supplement 4, pp. 11-19, 1998.

[16] M. N. Bucak, S. Sariözkan, P. B. Tuncer et al., "The effect of antioxidants on post-thawed Angora goat (Capra hircus ancryrensis) sperm parameters, lipid peroxidation and antioxidant activities," Small Ruminant Research, vol. 89, no. 1, pp. 24-30, 2010.

[17] S. C. Sikka, M. Rajasekaran, and W. J. G. Hellstrom, "Role of oxidative stress and antioxidants in male infertility," Journal of Andrology, vol. 16, no. 6, pp. 464-468, 1995.

[18] N. R. Desai, R. Mahfouz, R. Sharma, S. Gupta, and A. Agarwal, "Reactive oxygen species levels are independent of sperm concentration, motility, and abstinence in a normal, healthy, proven fertile man: a longitudinal study," Fertility and Sterility, vol. 94, no. 4, pp. 1541-1543, 2010.

[19] J. C. Kefer, A. Agarwal, and E. Sabanegh, "Role of antioxidants in the treatment of male infertility," International Journal of Urology, vol. 16, no. 5, pp. 449-457, 2009.

[20] D. Sanocka and M. Kurpisz, "Reactive oxygen species and sperm cells," Reproductive Biology and Endocrinology, vol. 2, pp. 12-26, 2004.

[21] F. R. Ochsendorf, "Infections in the male genital tract and reactive oxygen species," Human Reproduction Update, vol. 5, no. 5, pp. 399-420, 1999.

[22] A. Agarwal, K. P. Nallella, S. S. R. Allamaneni, and T. M. Said, "Role of antioxidants in treatment of male infertility: an overview of the literature," Reproductive BioMedicine Online, vol. 8, no. 6, pp. 616-627, 2004.

[23] S. G. Rhee, " $\mathrm{H}_{2} \mathrm{O}_{2}$, a necessary evil for cell signaling," Science, vol. 312, no. 5782, pp. 1882-1883, 2006.

[24] W. C. Ford, "Reactive oxygen species and sperm," Human Fertility, vol. 4, pp. 77-78, 2001.

[25] P. Guérin, S. El Mouatassim, and Y. Ménézo, "Oxidative stress and protection against reactive oxygen species in the pre-implantation embryo and its surroundings," Human Reproduction Update, vol. 7, no. 2, pp. 175-189, 2001.

[26] E. de Lamirande and C. Gagnon, "Impact of reactive oxygen species on spermatozoa: a balancing act between beneficial and detrimental effects," Human Reproduction, vol. 10, supplement 1, pp. 15-21, 1995.

[27] A. Agarwal, S. Gupta, and R. K. Sharma, "Role of oxidative stress in female reproduction," Reproductive Biology and Endocrinology, vol. 3, article 28, 2005.

[28] A. Agarwal and S. A. Prabakaran, "Mechanism, measurement, and prevention of oxidative stress in male reproductive physiology," Indian Journal of Experimental Biology, vol. 43, no. 11, pp. 963-974, 2005.
[29] S. C. Sikka, "Relative impact of oxidative stress on male reproductive function," Current Medicinal Chemistry, vol. 8, no. 7, pp. 851-862, 2001.

[30] V. Darley-Usmar, H. Wiseman, and B. Halliwell, "Nitric oxide and oxygen radicals: a question of balance," FEBS Letters, vol. 369, no. 2-3, pp. 131-135, 1995.

[31] M. Gavella and V. Lipovac, "NADH-dependent oxidoreductase (diaphorase) activity and isozyme pattern of sperm in infertile men," Archives of Andrology, vol. 28, no. 2, pp. 135141, 1992.

[32] S. Sariözkan, M. N. Bucak, P. B. Tuncer, P. A. Ulutaş, and A. Bilgen, "The influence of cysteine and taurine on microscopicoxidative stress parameters and fertilizing ability of bull semen following cryopreservation," Cryobiology, vol. 58, no. 2, pp. 134-138, 2009.

[33] N. Garrido, M. Meseguer, C. Simon et al., "Proxidative and antioxidative imbalance in human semen and its relation with male infertility," Asian Journal of Andrology, vol. 6, no. 1, pp. 59-65, 2004.

[34] R. J. Aitken and H. W. G. Baker, "Seminal leukocytes: passengers, terrorists or good Samaritans ?" Human Reproduction, vol. 10, no. 7, pp. 1736-1739, 1995.

[35] S. Shalika, K. Dugan, R. D. Smith, and S. L. Padilla, "The effect of positive semen bacterial and Ureaplasma cultures on invitro fertilization success," Human Reproduction, vol. 11, no. 12, pp. 2789-2792, 1996.

[36] R. J. Aitken, H. M. Fisher, N. Fulton et al., "Reactive oxygen species generation by human spermatozoa is induced by exogenous NADPH and inhibited by the flavoprotein inhibitors diphenylene iodonium and quinacrine," Molecular Reproduction and Development, vol. 47, no. 4, pp. 468-482, 1997.

[37] B. N. Hendin, P. N. Kolettis, R. K. Sharma, A. J. Thomas Jr., and A. Agarwal, "Varicocele is associated with elevated spermatozoal reactive oxygen species production and diminished seminal plasma antioxidant capacity," Journal of Urology, vol. 161, no. 6, pp. 1831-1834, 1999.

[38] F. F. Pasqualotto, R. K. Sharma, J. M. Potts, D. R. Nelson, A. J. Thomas Jr., and A. Agarwal, "Seminal oxidative stress in patients with chronic prostatitis," Urology, vol. 55, no. 6, pp. 881-885, 2000.

[39] R. K. Sharma, F. F. Pasqualotto, D. R. Nelson, A. J. Thomas Jr., and A. Agarwal, "Relationship between seminal white blood cell counts and oxidative stress in men treated at an infertility clinic," Journal of Andrology, vol. 22, no. 4, pp. 575-583, 2001.

[40] R. A. Saleh, A. Agarwal, E. Kandirali et al., "Leukocytospermia is associated with increased reactive oxygen species production by human spermatozoa," Fertility and Sterility, vol. 78, no. 6, pp. 1215-1224, 2002.

[41] F. Gonçalves, L. S.S. Barretto, R. P. Arruda, S. H.V. Perri, and G. Z. Mingoti, "Effect of antioxidants during bovine in vitro fertilization procedures on spermatozoa and embryo development," Reproduction in Domestic Animals, vol. 45, no. 1, pp. 129-135, 2010.

[42] N. Desai, R. Sharma, K. Maker, E. Sabnegh, and A. Agarwal, "Physiological and pathological levels of reactive oxygen species in neat semen of infertile men," Fertility and Sterility, vol. 92, pp. 1626-1631, 2009.

[43] H. Kodama, Y. Kuribayashi, and C. Gagnon, "Effect of sperm lipid peroxidation on fertilization," Journal of Andrology, vol. 17, no. 2, pp. 151-157, 1996. 
[44] E. de Lamirande and C. Gagnon, "A positive role for the superoxide anion in triggering hyperactivation and capacitation of human spermatozoa," International Journal of Andrology, vol. 16, no. 1, pp. 21-25, 1993.

[45] M. N. Bucak, A. Ateşşahin, and A. Yüce, "Effect of antioxidants and oxidative stress parameters on ram semen after the freeze-thawing process," Small Ruminant Research, vol. 75, no. 2-3, pp. 128-134, 2008.

[46] M. N. Bucak, A. Ateşşahin, O. Varişli, A. Yüce, N. Tekin, and A. Akçay, "The influence of trehalose, taurine, cysteamine and hyaluronan on ram semen. Microscopic and oxidative stress parameters after freeze-thawing process," Theriogenology, vol. 67, no. 5, pp. 1060-1067, 2007.

[47] A. K. Bansal and G. S. Bilaspuri, "Effect of ferrous ascorbate on in vitro capacitation and acrosome reaction in cattle bull spermatozoa," Animal Science Report, vol. 1, no. 2, pp. 69-77, 2007.

[48] B. Rao, J. C. Soufir, M. Martin, and G. David, "Lipid peroxidation in human spermatozoa as related to midpiece abnormalities and motility," Gamete Research, vol. 24, no. 2, pp. 127-134, 1989.

[49] A. K. Bansal and G. S. Bilaspuri, "Effect of ferrous sulphate and ascorbic acid on motility, viability and lipid peroxidation of crossbred cattle bull spermatozoa," Animal, vol. 2, no. 1, pp. 100-104, 2008.

[50] J. Twigg, N. Fulton, E. Gomez, D. S. Irvine, and R. J. Aitken, "Analysis of the impact of intracellular reactive oxygen species generation on the structural and functional integrity of human spermatozoa: lipid peroxidation, DNA fragmentation and effectiveness of antioxidants," Human Reproduction, vol. 13, no. 6, pp. 1429-1436, 1998.

[51] R. J. Aitken, J. S. Clarkson, and S. Fishel, "Generation of reactive oxygen species, lipid peroxidation, and human sperm function," Biology of Reproduction, vol. 41, no. 1, pp. 183-197, 1989.

[52] M. E. Gotz, A. Dirr, A. Freyberger, R. Burger, and P. Riederer, "The thiobarbituric acid assay reflects susceptibility to oxygen induced lipid peroxidation in vitro rather than levels of lipid hydroperoxides in vivo: a methodological approach," Neurochemistry International, vol. 22, no. 3, pp. 255-262, 1993.

[53] S. Chatterjee and C. Gagnon, "Production of reactive oxygen species by spermatozoa undergoing cooling, freezing, and thawing," Molecular Reproduction and Development, vol. 59, no. 4, pp. 451-458, 2001.

[54] Y. Wang, R. K. Sharma, and A. Agarwal, "Effect of cryopreservation and sperm concentration on lipid peroxidation in human semen," Urology, vol. 50, no. 3, pp. 409-413, 1997.

[55] C. O’Flaherty, M. Beconi, and N. Beorlegui, "Effect of natural antioxidants, superoxide dismutase and hydrogen peroxide on capacitation of frozen-thawed bull spermatozoa," Andrologia, vol. 29, no. 5, pp. 269-275, 1997.

[56] J. G. Kim and S. Parthasarathy, "Oxidation and the spermatozoa," Seminars in Reproductive Endocrinology, vol. 16, no. 4, pp. 235-239, 1998.

[57] A. K. Bansal and G. S. Bilaspuri, "Effect of manganese on bovine sperm motility, viability, and lipid peroxidation in vitro," Animal Reproduction CBRA, vol. 5, no. 3-4, pp. 90-96, 2008.

[58] J. K. Miller, E. Brzezinska-Slebodzinska, and F. C. Madsen, "Oxidative stress, antioxidants, and animal function," Journal of Dairy Science, vol. 76, no. 9, pp. 2812-2823, 1993.

[59] H. Kumar and S. Mahmood, "The use of fast acting antioxidants for the reduction of cow placental retention and subsequent endometritis," Indian Journal of Animal Sciences, vol. 71, no. 7, pp. 650-653, 2001.
[60] A. K. Bansal and G. S. Bilaspuri, "Antioxidant effect of vitamin E on motility, viability and lipid peroxidation of cattle spermatozoa under oxidative stress," Animal Science Papers and Reports, vol. 27, no. 1, pp. 5-14, 2009.

[61] G. S. Bilaspuri and A. K. Bansal, " $\mathrm{Mn}^{2+}$ : a potent antioxidant and stimulator of sperm capacitation and acrosome reaction in crossbred cattle bulls," Archiv fur Tierzucht, vol. 51, no. 2, pp. 149-156, 2008.

[62] A. K. Bansal and G. S. Bilaspuri, "Oxidative stress alters membrane sulfhydryl status, lipid and phospholipid contents of crossbred cattle bull spermatozoa," Animal Reproduction Science, vol. 104, no. 2-4, pp. 398-404, 2008.

[63] M. N. Bucak, P. B. Tuncer, S. Sariözkan, and P. A. Ulutaş, "Comparison of the effects of glutamine and an amino acid solution on post-thawed ram sperm parameters, lipid peroxidation and anti-oxidant activities," Small Ruminant Research, vol. 81, no. 1, pp. 13-17, 2009.

[64] O. Uysal and M. N. Bucak, "Effects of oxidized glutathione, bovine serum albumin, cysteine and lycopene on the quality of frozen-thawed ram semen," Acta Veterinaria Brno, vol. 76, no. 3, pp. 383-390, 2007.

[65] N. S .S. Reddy, G. J. Mohanarao, and S. K. Atreja, "Effects of adding taurine and trehalose to a tris-based egg yolk extender on buffalo(Bubalua bubalis) sperm quality following cryopreservation," Animal Reproduction Science, vol. 119, pp. 183-190, 2010.

[66] J. J. Tarín, J. Brines, and A. Cano, "Is antioxidant therapy a promising strategy to improve human reproduction?" Human Reproduction, vol. 13, no. 6, pp. 1415-1424, 1998.

[67] M. I. Yousef, G. A. Abdallah, and K. I. Kamel, "Effect of ascorbic acid and Vitamin E supplementation on semen quality and biochemical parameters of male rabbits," Animal Reproduction Science, vol. 76, no. 1-2, pp. 99-111, 2003.

[68] R. S. Cheema, A. K. Bansal, and G. S. Bilaspuri, "Manganese provides antioxidant protection for sperm cryopreservation that may offer new consideration for clinical fertility," Oxidative Medicine and Cellular Longevity, vol. 2, no. 3, pp. 152-159, 2009.

[69] T. M. Said, S. Grunewald, U. Paasch, M. Rasch, A. Agarwal, and H.-J. Glander, "Effects of magnetic-activated cell sorting on sperm motility and cryosurvival rates," Fertility and Sterility, vol. 83, no. 5, pp. 1442-1446, 2005.

[70] S. Chatterjee, E. De Lamirande, and C. Gagnon, "Cryopreservation alters membrane sulfhydryl status of bull spermatozoa: protection by oxidized glutathione," Molecular Reproduction and Development, vol. 60, no. 4, pp. 498-506, 2001. 

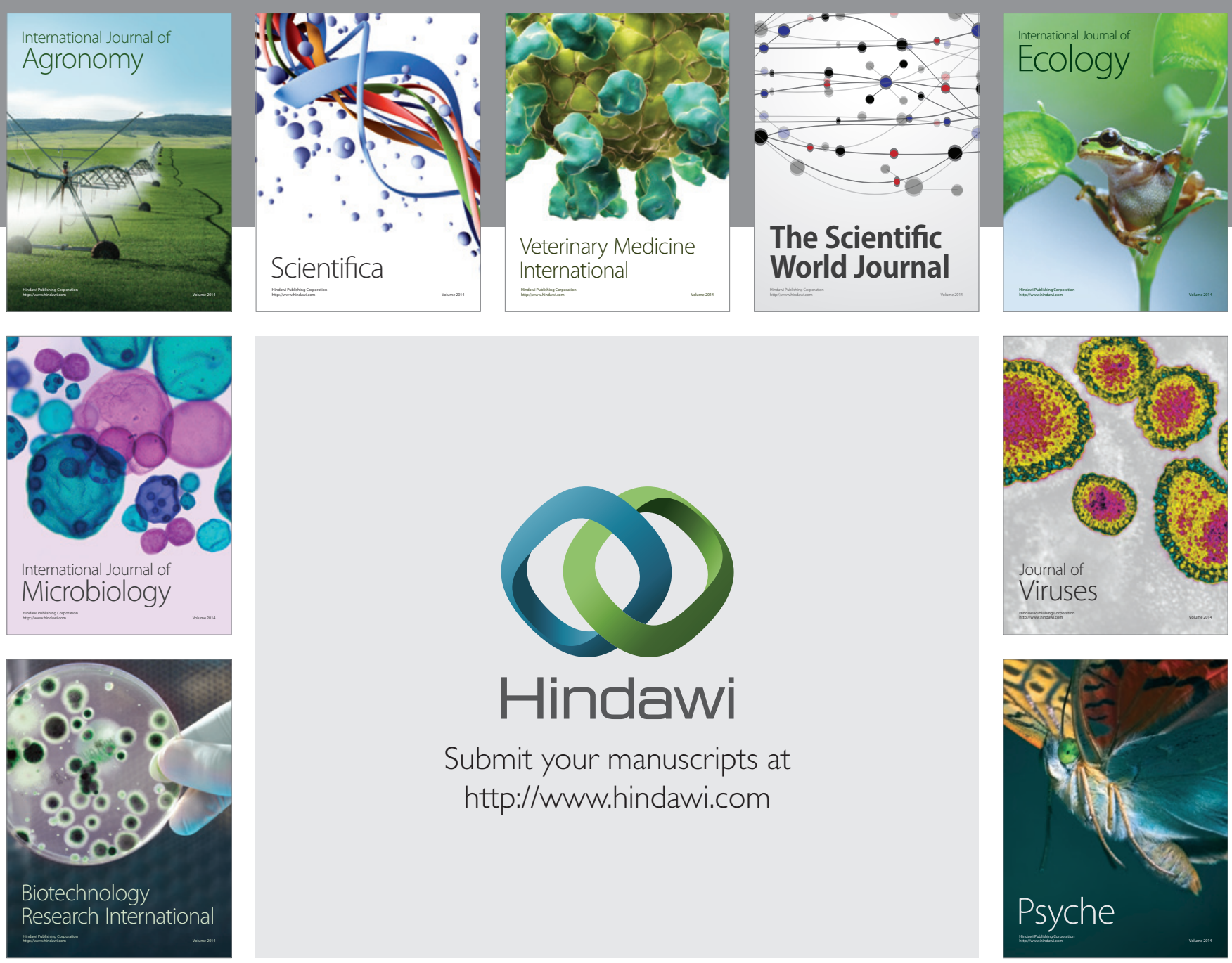

Submit your manuscripts at

http://www.hindawi.com
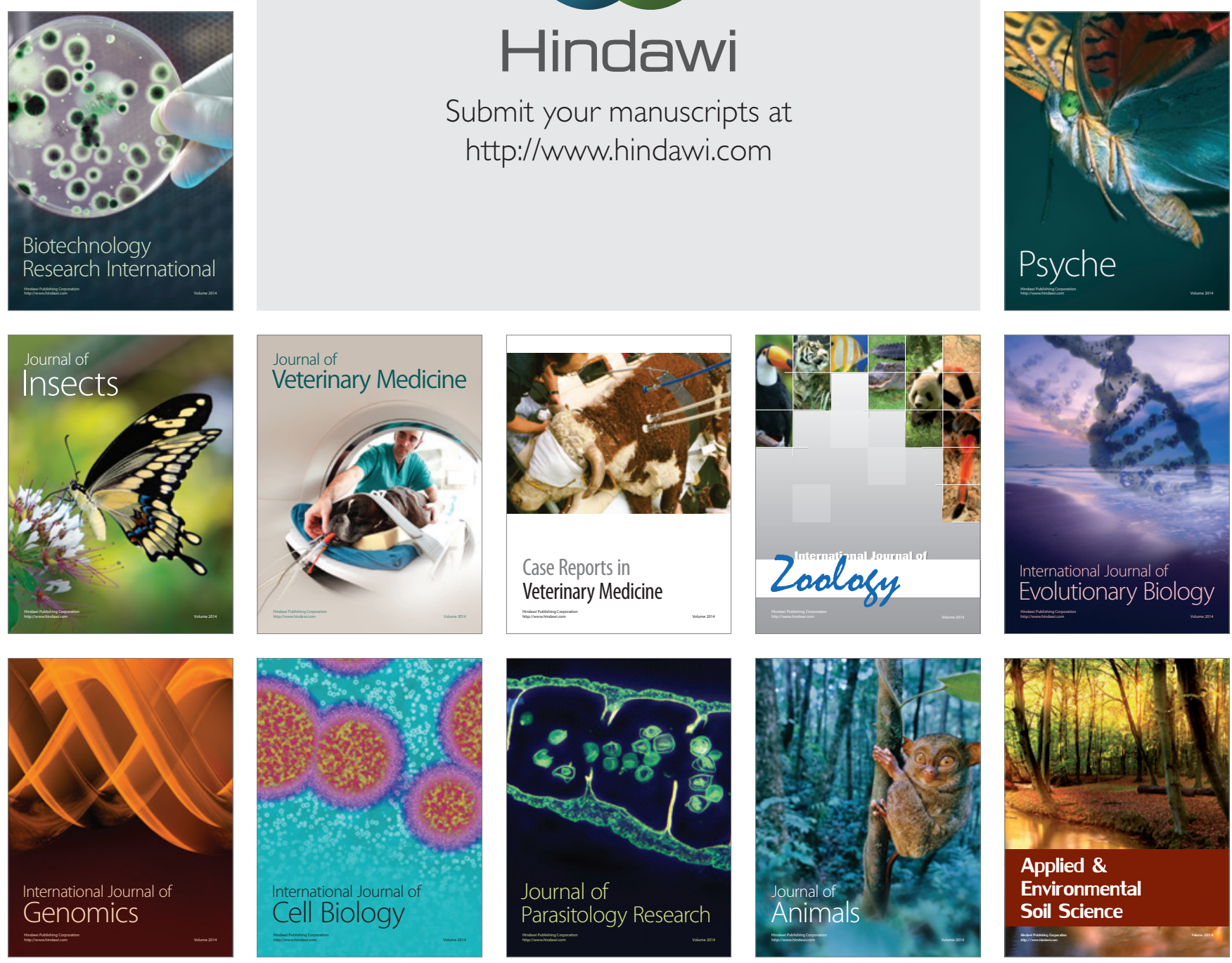